\title{
Role of liver atrophy, hepatic resection and hepatocyte hyperplasia in the development of portal hypertension in biliary disease
}

\author{
N S HADJIS AND L H BLUMGART \\ From the Hepatobiliary Surgical Unit, Royal Postgraduate Medical School, Hammersmith Hospital, London
}

SUMMARY Portal fibrosis is considered to be pivotal in the pathogenesis of portal hypertension associated with extrahepatic biliary obstruction. The histological features, however, include diffuse hepatocyte hyperplasia as well as portal fibrosis, but not cirrhosis, and it is possible that the contribution of hepatocyte hyperplasia in the initiation of portal hypertension is equally important. If so, we hypothesised that patients with biliary obstruction and a coincident condition such as liver atrophy, or hepatic resection, with the potential of accelerating the hepatocyte proliferation caused by biliary obstruction itself, might be expected to develop portal hypertension earlier than patients with biliary obstruction alone. To examine this concept we studied 10 patients with postcholecystectomy bile duct stricture, portal hypertension and liver atrophy, or hepatic resection (group I) and compared them with nine patients with postcholecystectomy stricture and portal hypertension, but no atrophy or resection (group II). Portal hypertension was diagnosed a mean 28 months (range 18-48 months) after cholecystectomy in group I compared with 62 months (range 36-100 months) for patients in group II $(\mathrm{p}<0.005$ Mann-Whitney test). Thus hepatocyte hyperplasia may be an important part of the mechanism underlying the development of portal hypertension in chronic biliary disease.

Diffuse hepatocyte hyperplasia and extensive portal fibrosis, but not cirrhosis, are the histological changes observed in most cases of portal hypertension associated with chronic bile duct obstruction.' Many reports have emphasised that the onset of portal hypertension is primarily the result of the early development of fibrosis. ${ }^{2-4}$ The contribution of hepatocyte hyperplasia, however, may also be important and it is possible that in some cases portal hypertension is initiated by bile duct obstruction and a concomitant condition that has accelerated the hepatocyte hyperplasia induced by biliary obstruction itself. Patients with such disease might be at risk of developing portal hypertension at an earlier stage than patients with biliary obstruction alone.

Address for correspondence: Mr N S Hadjis, FRCS, RPMS, Hammersmith Hospital, Ducane Road, London W12 0HS

Received for publication 23 January, 1987.
A significant number of patients with benign high biliary obstruction have liver atrophy, ${ }^{5}$ which has been shown experimentally to be a potent stimulus of hepatocyte hyperplasia. ${ }^{6}$ If hepatocyte hyperplasia is important in the pathogenesis of portal hypertension, such patients might be expected to develop portal hypertension more frequently and more quickly than patients with obstruction but without hepatocyte hyperplasia associated with atrophy. Furthermore, as hepatocyte hyperplasia always follows hepatic resection, bile duct obstruction occurring in patients after liver resection might also be associated with an increased risk of portal hypertension. Corroboration of this hypothesis would imply that patients with bile duct stricture and either liver atrophy or hepatic resection require early surgery, irrespective of the degree of obstruction, to improve bile flow and reduce the hepatocytic hyperplasia and portal fibrosis induced by biliary obstruction. 
To examine this hypothesis we compared the duration of disease in patients with bile duct obstruction, portal hypertension and liver atrophy or hepatic resection, with that in patients with bile duct obstruction and portal hypertension but no obvious atrophy and with patients with bile duct obstruction and atrophy but no portal hypertension.

\section{Methods}

\section{PATIENTS}

Of 108 patients admitted to the Hepatobiliary Unit at Hammersmith Hospital with chronic biliary obstruction caused by a postcholecystectomy bile duct stricture, 24 had associated portal hypertension or liver atrophy, or both. These 24 patients are the subject of this study.

The Table shows the symptoms with which the patients had been referred. The diagnosis of obstruction was made on percutaneous transhepatic cholangiography. In 22 patients both lobes were obstructed but two patients had unilobar obstruction. Liver atrophy (defined as a reduction in size of a lobe or of a recognised anatomical segment by at least an estimated $50 \%$ ) was diagnosed on the basis of findings demonstrated on cholangiography, computed tomography, ${ }^{8}$ scintigraphy and selective coeliac angiography with indirect portography ${ }^{5}$ (Figure). Liver biopsy specimens were obtained in 22 patients at the end of a surgical procedure. Depending on the presence of portal hypertension or atrophy, or both, three groups were studied:

Group I comprised 10 patients (seven men and three women, mean age 45 years) with bile duct obstruction, portal hypertension, and lobar liver atrophy (nine cases) or bile duct obstruction, portal hypertension and previous partial hepatectomy (one case). This patient had undergone right lobectomy in a previous attempt to repair a high bile duct stricture which subsequently recurred.

Group II consisted of nine patients (four men and five women, mean age 52 years) with bile duct obstruction and portal hypertension but without obvious atrophy.

Group III contained five patients (two men and three women, mean age 55 years) with bile duct obstruction and lobar atrophy (four cases) or segmental atrophy (one case) but no portal hypertension. These patients had been investigated in a manner comparable with those in groups I and II but only three underwent repair of bile duct stricture; the other two had only unilateral hepatic duct obstruction and were treated conservatively.

The criteria on which portal hypertension was diagnosed were as follows: group I: raised portal pressure (measured at surgery by cannulation of a
Table Symptoms and signs with which patients were referred to Hammersmith Hospital, analysed according to their study group (see methods)

\begin{tabular}{llll}
\hline Patients & $\begin{array}{l}\text { Jaundicel } \\
\text { cholangitis }\end{array}$ & $\begin{array}{l}\text { Oesophageal } \\
\text { varices }\end{array}$ & Haematemesis \\
\hline Group I (no 10) & & & + \\
2 & + & + & - \\
1 & + & + & - \\
7 & + & - & + \\
Group II (no 9) & & + & - \\
3 & + & - & - \\
6 & + & - & \\
Group III (no 5) & & & \\
5 & + & & \\
\hline
\end{tabular}

branch of the portal venous system) in three patients, including two with varices, at $32 \mathrm{~mm}, 36 \mathrm{~mm}$, and 33 $\mathrm{mmHg}$, respectively; oesophageal varices in three (endoscopy three, angiography two); and splenomegaly in four patients (CT scan four, angiography three, physical examination two). Group II: raised portal pressure in two patients (one with varices) at $35 \mathrm{~mm}$ and $32 \mathrm{mmHg}$, respectively; oesophageal varices in three (endoscopy three, angiography one); and splenomegaly in four patients (CT scan four, angiography two, physical examination three). In five patients with splenomegaly (two from group I and three from group II) splenic tissue became available at laparotomy or necropsy and microscopical changes associated with portal hypertension (white pulp atrophy, increased fibrosis and iron deposition) were observed in all.

The strictures of the 22 patients with bilateral lobe obstruction were graded according to the Bismuth classification based on the cholangiographic appearance of the stricture:" Bismuth grade I: 0 patients; Bismuth grade II: three patients (group I: 0, group II: one, group III: two); Bismuth grade III: 11 patients (group I: five, group II: five, group III: one); Bismuth grade IV: eight patients (group I: five, group II: three).

The mean number of surgical procedures done before the diagnosis of portal hypertension was reached was 2.3 in group I and 2.8 in group II. The corresponding figure for group III is two. The frequency of cholangitic episodes and the treatment instituted were similar.

The duration of disease was considered to be the time since an operative ductal injury had been recorded during cholecystectomy up to the time portal hypertension was firstly diagnosed. Because the ductal injury that eventually caused atrophy may not have always been inflicted during the operation of cholecystectomy but at a later procedure undertaken to correct a formed stricture, it is likely that the interval between the development of atrophy and the 


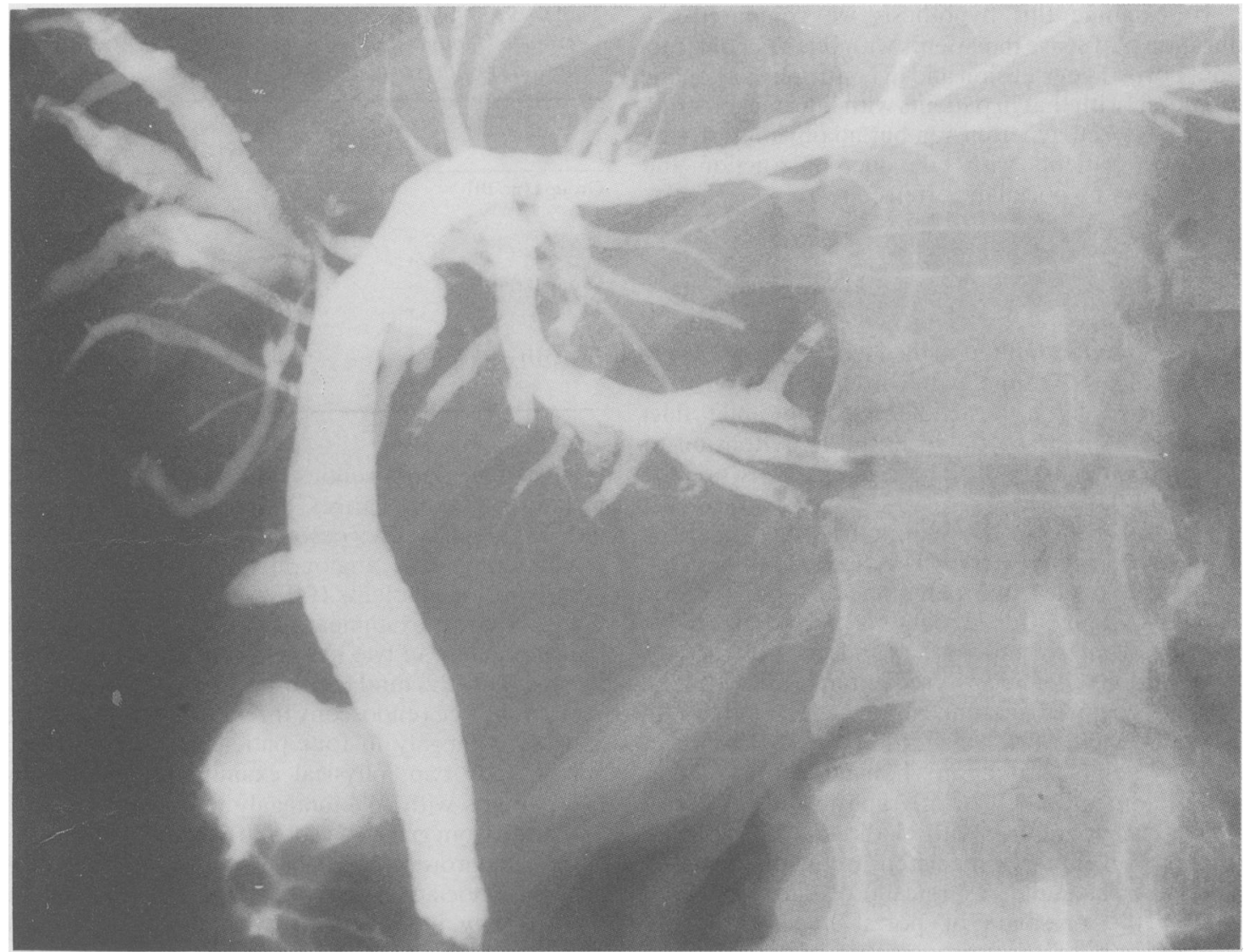

Fig. 1a

Figure Patient with postcholecystectomy high bile duct stricture. Portal hypertension, with oesophageal varices, was diagnosed 18 months after cholecystectomy. (a) Percutaneous cholangiogram showing crowding of intrahepatic ducts in a small right lobe and dilated ducts within a hypertrophied left lobe.
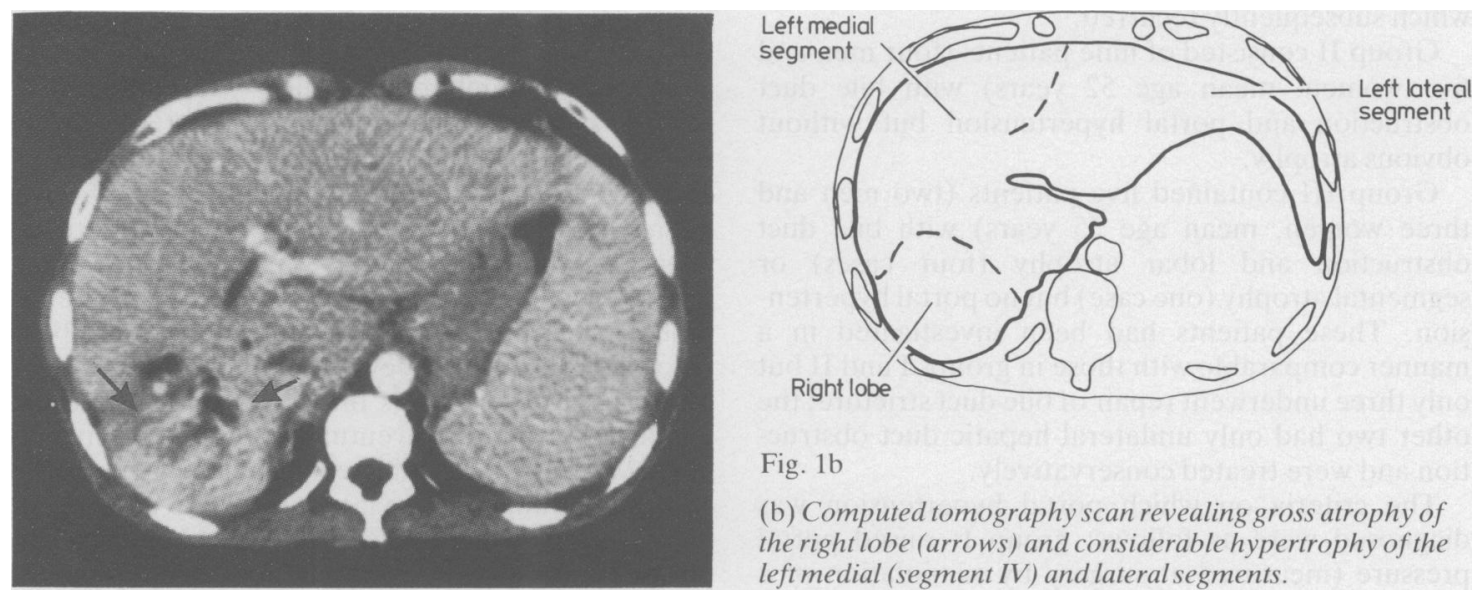

Fig. 1b

(b) Computed tomography scan revealing gross atrophy of the right lobe (arrows) and considerable hypertrophy of the left medial (segment IV) and lateral segments. 


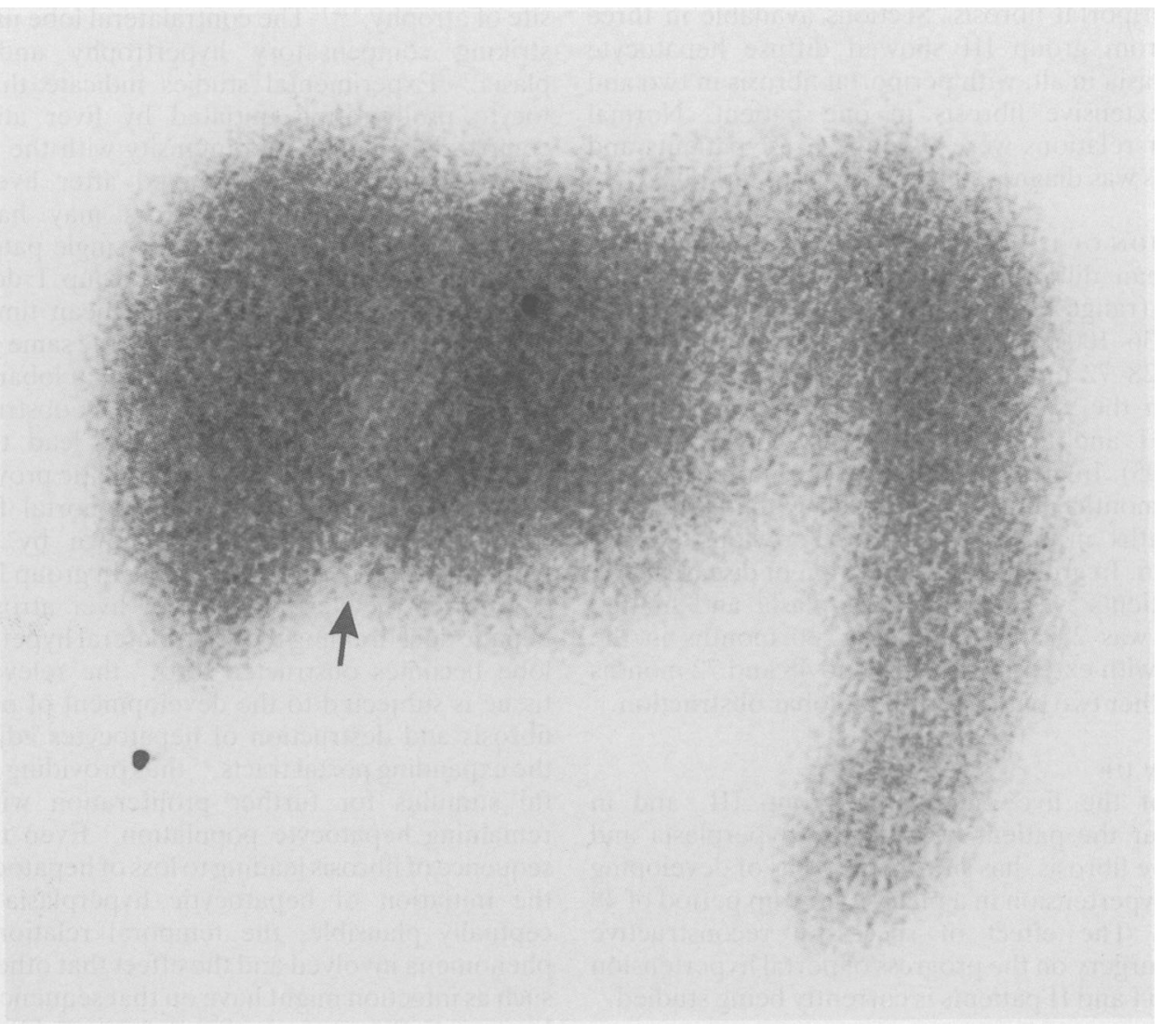

Fig. 1c

Figure continued (c) Colloid liver scan. Note absence of radionuclide uptake by the atrophied right lobe. There is pronounced hypertrophy of the quadrate lobe and left lateral segment, separated by the umbilical fissure (arrow). Marked enlargement of spleen.

onset of portal hypertension is overestimated by this definition. For group III the duration of disease was estimated from the date of cholecystectomy up to the time of investigation at Hammersmith Hospital.

The extent of fibrosis, the zonal distribution of hepatocyte hyperplasia and its lobular or nodular pattern were defined according to criteria reviewed recently.' Retained vascular relations - that is, identification of portal tracts and hepatic venous radicles in appropriate relation to each other, excluded the diagnosis of cirrhosis. ${ }^{\prime}$

\section{STATISTICAL ANALYSIS}

Mann-Whitney test was applied.

\section{Results}

\section{OPERATIVE FINDINGS}

The diagnosis of atrophy was confirmed in each of the 12 patients who underwent surgery. Examination of detailed operative notes on the degree of atrophy showed no striking difference between cases in groups I and III except for the patient (group III) with segmental atrophy. (Radiological assessment of atrophy in the two patients treated conservatively showed it to be comparable with the other cases).

\section{ANGIOGRAPHIC FINDINGS}

Angiographic information was available in seven patients from group I, five from group II and two patients from group III; narrowing or occlusion of the main right or left branch of the portal vein was diagnosed in three, two, and one patients, respectively.

\section{PATHOLOGICAL FINDINGS}

Microscopic examination of sections from all patients in groups I and II showed diffuse hepatocyte hyperplasia and extensive fibrosis with the exception of one patient (group I) who had diffuse hyperplasia but 
only periportal fibrosis. Sections available in three cases from group III showed diffuse hepatocyte hyperplasia in all, with periportal fibrosis in two and more extensive fibrosis in one patient. Normal vascular relations were retained in 21 patients and cirrhosis was diagnosed in one case (group II).

DURATION OF DISEASE

The mean duration and range of disease was 28 months (range 18-48 months) in group I, 62 months (range 36-100 months) in group II and 42 months (range 28-72 months) in group III. The difference between the two groups with portal hypertension (group I and group II) is statistically significant $(\mathrm{p}<0 \cdot 005)$. In group I, the mean duration of disease was 28 months in the nine patients with atrophy and 24 months in the patient with previous hepatic resection. In group III, the duration of disease in the two patients with diffuse hyperplasia and limited fibrosis was 28 and 32 months, 30 months in the patient with extensive fibrosis and 48 and 72 months in the other two patients with unilobar obstruction.

\section{FOLLOW UP}

None of the five patients in group III, and in particular the patient with diffuse hyperplasia and extensive fibrosis, has shown any signs of developing portal hypertension in a mean follow up period of 48 months. The effect of successful reconstructive biliary surgery on the progress of portal hypertension in group I and II patients is currently being studied.

\section{Discussion}

Portal hypertension develops in about $20 \%$ of patients with chronic bile duct obstruction and has been associated with a bad prognosis. ${ }^{10-12}$ The 30 day hospital mortality in 11 such patients reported recently was $36 \%$ compared with $3 \cdot 2 \%$ for patients with comparable bile duct obstruction but without portal hypertension." ${ }^{10}$ Although many risk factors have been identified for the development of portal hypertension, including a protracted course of obstruction and frequent episodes of cholangitis, ${ }^{1012}$ their contribution to the structural changes occurring in the liver has not been analysed. This may reflect the prevailing notion that the mechanism of obstruction to the portal flow is the same as that which has been postulated for the more common forms of hepatic cirrhosis - that is, the existence of hyperplastic nodules. ${ }^{213-15}$ It is worth noting, however, that hepatocyte hyperplasia in portal hypertension consequent on large duct obstruction is distinctly lobular rather than nodular in pattern. ${ }^{1}$

Lobar atrophy of the liver usually signifies hepatic duct obstruction, or portal venous occlusion, to the site of atrophy. ${ }^{516} 17$ The contralateral lobe undergoes striking compensatory hypertrophy and hyperplasia. ${ }^{16}$ Experimental studies indicate that hepatocyte proliferation initiated by liver atrophy is comparable in time and intensity with the adaptive hyperplastic response observed after liver resection. ${ }^{618}$ Although other factors may have contributed, it is interesting that the single patient with previous hepatic lobectomy in group I developed portal hypertension close to the mean time of the other nine patients with atrophy in the same group. It is perhaps worth noting, however, that lobar atrophy consequent on unilateral hepatic duct obstruction or portal venous occlusion does not lead to portal hypertension and can be asymptomatic provided the contralateral lobe enjoys normal portal flow and normal biliary drainage ${ }^{19}$ as shown by the two patients with unilobar obstruction in group III.

If, after the development of liver atrophy, the hepatic duct draining the contralateral hypertrophied lobe becomes obstructed also, ${ }^{17}$ the relevant liver tissue is subjected to the development of periportal fibrosis and destruction of hepatocytes adjacent to the expanding portal tracts, ${ }^{12}$ thus providing a powerful stimulus for further proliferation within the remaining hepatocyte population. ${ }^{5}$ Even though a sequence of fibrosis leading to loss of hepatocytes and the initiation of hepatocytic hyperplasia is conceptually plausible, the temporal relation of the phenomena involved and the effect that other factors such as infection might have on that sequence are not known. Suffice to say that extensive fibrosis and progression to biliary cirrhosis occurs regularly in experimental bile duct obstruction in the absence of infection. ${ }^{20}$ Many clinical studies have shown a correlation between the frequency of cholangitic episodes and the subsequent development of portal hypertension ${ }^{12}{ }^{21}$ but in one study the inverse correlation was observed between cholangitis and biliary cirrhosis. ${ }^{14}$ Moreover, the fibrosis of uncomplicated ductal obstruction cannot be discriminated from the fibrosis of 'infected biliary cirrhosis'. ${ }^{22}$

The average time of five years taken for portal hypertension to develop in patients without atrophy (group II) is in accord with reported figures." 12 Although the onset of portal hypertension under two years in some cases of bile duct obstruction has been noted by others, no causative factors were identified and no conclusions were drawn as to the operative mechanisms. ${ }^{12}$ The results of this study suggest that the contribution of hepatocyte hyperplasia to the development of portal hypertension in chronic biliary obstruction is substantial and important. While bile duct obstruction effects a degree of periportal hepatocyte hyperplasia which may become diffuse if the obstruction is unrelieved, ${ }^{\prime}$ an early generation of 
hepatocyte hyperplasia by an appropriate stimulus may considerably shorten the time required for hypertension to develop.

It is of interest that one patient in group I had portal hypertension in the presence of limited periportal fibrosis as assessed in sections from a sizeable piece of liver removed to facilitate surgical access. Although we have not encountered a case of extrahepatic duct obstruction and portal hypertension not associated with diffuse hepatocyte hyperplasia, it may be that extensive periportal fibrosis is not essential for hypertension to develop. On the other hand, an explanation for the absence of demonstrable portal hypertension in three patients with diffuse hyperplasia in group III is not obvious although it is noted that two of them had only limited fibrosis. Portal hypertension was diagnosed on clinical and imaging signs generally regarded as indicating a raised portal pressure but it has been remarked that their absence does not exclude a high portal pressure in extrahepatic biliary disease. 112 Furthermore, our hypothesis predicts an increased risk rather than a certainty of early portal hypertension and it may be pertinent to speculate that had these three patients not been submitted to successful biliary drainage at around 30 months after cholecystectomy, with the expected resolution of fibrosis and the arrest of further hepatocyte proliferation, they might still have developed portal hypertension within the time range of patients with atrophy in group I, or, at any rate, earlier than patients without atrophy in group II. Thus in the light of this and other recent work ${ }^{1}$ it seems that both component processes are important and it may be untenable to view portal fibrosis as the critical factor in the pathogenesis of portal hypertension in chronic bile duct disease without reference to hepatocyte hyperplasia. ${ }^{4}$

Progression of the hepatic disease and the development of oesophageal varices which have been noted in some patients with biliary obstruction after restoration of bile flow ${ }^{4114}$ is disturbing and not readily understood, although such deterioration may not be unexpected with established true cirrhosis, incomplete biliary decompression, ${ }^{414}$ or extrahepatic portal venous occlusion. "Indeed, the significance of the observation that most patients with obstructive biliary disease and portal hypertension have no cirrhosis ${ }^{1}$ rests with the distinct possibility of regression of structural changes in the liver including fibrosis consequent upon successful biliary drainage..$^{2324}$ The recommended practice of early and efficient relief of bile duct obstruction ${ }^{4}$ cannot be overemphasised in cases where a coincident disease causing hepatocyte proliferation is in operation.

Finally, our hypothesis may also explain the pathogenesis of portal hypertension that occasionally develops after relief of biliary obstruction that has necessitated liver resection, a sequence that we observed in one of 16 patients with hilar cholangiocarcinoma undergoing hepatic resection. Investigations revealed patency of the ductal anastomosis and vascular channels, and it is possible that a slower than normal resolution of extensive portal fibrosis secondary to obstruction in association with the development of diffuse hepatocyte hyperplasia consequent on resection account for the outcome in this patient. Experimental data show early return of portal pressure and liver perfusion to normal after hepatic resection $^{25}$ and although acute portal hypertension has been reported to occur after hepatic resection in patients without bile duct disease, ${ }^{26}$ this has not been confirmed in other series ${ }^{27}$ and we have not encountered it in 68 major resections with the exception of one case complicated by portal venous occlusion.

We are indebted to Professor $\mathrm{K}$ Weinbren for reporting on the sections. We acknowledge the considerable help of our colleagues, particularly Professor D Allison in the Department of Diagnostic Radiology. We also thank Mr W Trubridge of the Department of Medical Illustration at Hammersmith Hospital and Miss H Skelton for her secretarial assistance.

\section{References}

1 Weinbren K, Hadjis NS, Blumgart LH. Structural aspects of the liver in patients with biliary disease and portal hypertension. J Clin Pathol 1985; 38: 1013-20.

2 Desmet VJ. Cholestasis; extrahepatic obstruction and secondary biliary cirrhosis. In: MacSween RNM, Anthony PP, Scheuer PJ, eds. Pathology of the liver. Edinburgh: Churchill Livingstone, 1979: 272-305.

3 Shorter RG, Baggenstoss AH. Extrahepatic cholestasis. Chronology of histologic changes in the liver. Am J Clin Pathol 1959; 32: 10-17.

4 Franco D, Gigou M, Szekely A-M, Bismuth H. Portal hypertension after bile duct obstruction. Arch Surg 1979; 114: 1064-7.

5 Hadjis NS, Blumgart LH. Liver hyperplasia, hypertrophy and atrophy; clinical relevance. In: Blumgart LH, ed. Surgery of the liver and biliary tract. Edinburgh: Churchill Livingstone. (In press).

6 Weinbren $\mathrm{K}$, Tarsh E. The mitotic response in the rat liver after different regenerative stimuli. BrJ Exp Pathol 1964; 45: 475-80.

7 Myracle MR, Stadalnik RC, Blaisdell FW, Farkas JP, Matin P. Segmental biliary obstruction: diagnostic significance of bile duct crowding. $A J R$ 1981; 137: 169-71.

8 Carr D, Hadjis NS, Banks L, Hemingway A, Blumgart 
LH. Computed tomography of hilar cholangiocarcinoma. $A J R$ 1985; 145: 53-6.

9 Bismuth H. Postoperative strictures of the bile duct. In: Blumgart LH, ed. The biliary tract. Clinical Surgery International Vol. 5. Edinburgh: Churchill Livingstone, 1982: 209-18.

10 Blumgart LH, Kelley CJ, Benjamin IS. Benign bile duct stricture following cholecystectomy. Critical factors in management. Br J Surg 1984; 71: 836-43.

11 Adson MA, Wychulis AR. Portal hypertension in secondary biliary cirrhosis. Arch Surg 1968; 96: 604-12.

12 Sedgwick CE, Poulantzas JK, Kune GA. Management of portal hypertension secondary to bile duct strictures. Ann Surg 1966; 163: 949-53.

13 Kelty RH, Baggenstoss AH, Butt HR. The relation of the regenerated liver nodule to the vascular bed in cirrhosis. Gastroenterology 1950; 15: 285-95.

14 Scobie BA, Summerskill WHJ. Hepatic cirrhosis secondary to obstruction of the biliary system. Am J Dig Dis 1965; 10: 135-46.

15 Doehlert CA, Baggenstoss AH, Cain JC. Obstructive biliary cirrhosis and alcoholic cirrhosis. Am J Clin Pathol 1955; 25: 902-14.

16 Rous P, Larimore LD. Relation of the portal blood to liver maintenance. J Exp Med 1920; 31: 609-32.

17 Marshall JM. Tumors of the bile ducts. Surg Gynecol Obstet 1932; 54: 6-12.

18 Lawrence W, Joly D, Brasfield R. A comparative study of various mechanisms of hepatic restoration in the rat. Surgery 1959; 45: 543-51.

19 Hadjis NS, Carr D, Banks LM, Gibson R, Blumgart LH. Expectant management of patients with unilateral hepatic duct obstruction and liver atrophy. Gut 1986; 27: 1223-7.

20 Richardson ML. Biliary cirrhosis in the rabbit. $J$ Exp Med 1911; 14: 401-7.

21 Ekman C-A, Sandblom P. Bilio-intestinal anastomosis as a cause of liver cirrhosis with portal hypertension. Acta Chir Scand 1962; 123: 383-8.

22 MacMahon HE. Biliary cirrhosis. Lab Invest 1955; 4: 243-61.

23 Thaler MM, Gellis SS. Studies in neonatal hepatitis and biliary atresia. Am J Dis Child 1968; 116: 271-9.

24 Bunton GL, Cameron R. Regeneration of liver after biliary cirrhosis. Ann NY Acad Sci 1963; 111: 412-21.

25 Rice GC, Leiberman DP, Mathie RT, Ryan CJ, Harper AM, Blumgart LH. Liver tissue blood flow measured by $K R$ clearance in the anaesthetized rat before and after partial hepatectomy. Br J Exp Pathol 1977; 58: 243-50.

26 Stone HH, Long WD, Smith RB, Physiologic considerations in major hepatic resections. Am J Surg 1969; 117: 78-84.

27 Buerk CA, Putnam CW, Starzl TE. Major hepatic resection and portal pressure. Surg Gynecol Obstet 1977; 144: 853-4. 